\title{
Early intervention with gastrodin reduces striatal neurotoxicity in adult rats with experimentally-induced diabetes mellitus
}

\author{
YU-HAN QI ${ }^{1,2^{*}}$, RUI ZHU ${ }^{1,2^{*}}$, QING WANG ${ }^{1,3}$, QIAN LI $^{1,2}$, YI-DAN LIU $^{4}$, \\ ZHONG-YI QIAN ${ }^{5}$, ZHI-HONG YANG ${ }^{1}$, ZHI-HAO MU ${ }^{1}$, XIN-JIE LIU ${ }^{1,3}$, MEI-YAN ZHANG ${ }^{1}$, \\ $\mathrm{XIE} \mathrm{WANG}^{1}$, XIN-YU LIAO ${ }^{1}$, QI WAN $^{6}$, DI LU ${ }^{7}$ and YING-YING ZOU ${ }^{1}$ \\ ${ }^{1}$ Department of Pathology and Pathophysiology, Faculty of Basic Medical Sciences, Kunming Medical University; \\ ${ }^{2}$ The First Faculty of Clinical Medicine, Kunming Medical University; ${ }^{3}$ The Second Faculty of Clinical Medicine, \\ Kunming Medical University; ${ }^{4}$ Institute of Drug Discovery and Development, Kunming Pharmaceutical Corporation; \\ ${ }^{5}$ Department of Morphological Laboratory, Faculty of Basic Medical Sciences, Kunming Medical University, \\ Kunming, Yunnan 650500; ${ }^{6}$ Institute of Neuroregeneration and Neurorehabilitation, Department of Neurosurgery \\ of The Affiliated Hospital, Qingdao University, Qingdao, Shandong 266071; ${ }^{7}$ Biomedical Engineering Research Center, \\ Kunming Medical University, Kunming, Yunnan 650500, P.R. China
}

Received June 28, 2018; Accepted January 11, 2019

DOI: $10.3892 / \mathrm{mmr} .2019 .9954$

\begin{abstract}
Glutamate-induced excitotoxicity in the striatum has an important role in neurodegenerative diseases. It has been reported that diabetes mellitus (DM) induces excitotoxicity in striatal neurons, although the underlying mechanism remains to be fully elucidated. The present study aimed to investigate the effect of gastrodin on DM-induced excitotoxicity in the striatal neurons of diabetic rats. Adult Sprague-Dawley rats were divided into control, diabetic, and gastrodin intervention groups. Diabetes in the rats was induced with a single intraperitoneal injection of streptozotocin $(65 \mathrm{mg} / \mathrm{kg})$. In the gastrodin groups, the rats were gavaged with 60 or $120 \mathrm{mg} / \mathrm{kg} /$ day gastrodin for 6 weeks, 3 weeks following the induction of diabetes. Pathological alterations in the striatum were assessed using hematoxylin and eosin (H\&E) staining. The protein expression levels of phosphorylated (p)-extracellular signal-regulated kinase (ERK)1/2, p-mitogen-activated protein kinase kinase (MEK)1/2, tyrosine receptor kinase B (TrKB) and brain-derived neurotrophic factor (BDNF) in the
\end{abstract}

Correspondence to: Professor Ying-Ying Zou, Department of Pathology and Pathophysiology, Faculty of Basic Medical Sciences, Kunming Medical University, 1168 West Chunrong Road, Kunming, Yunnan 650500, P.R. China

E-mail: zouyingying@kmmu.edu.cn

Professor Di Lu, Biomedical Engineering Research Center, Kunming Medical University, 1168 West Chunrong Road, Kunming, Yunnan 650500, P.R. China

E-mail: ludi@kmmu.edu.cn

*Contributed equally

Key words: diabetes, striatum, neurons, excitotoxicity, gastrodin striatal neurons were evaluated by western blotting and double immunofluorescence. Additionally, the extracellular levels of glutamate were measured by microanalysis followed by high-pressure-liquid-chromatography. In diabetic rats, striatal neuronal degeneration was evident following H\&E staining, which revealed the common occurrence of pyknotic nuclei. This was coupled with an increase in glutamate levels in the striatal tissues. The protein expression levels of p-ERK1/2, p-MEK1/2, TrKB and BDNF in the striatal tissues were significantly increased in the diabetic rats compared with those in the normal rats. In the gastrodin groups, degeneration of the striatal neurons was ameliorated. Furthermore, the expression levels of glutamate, p-ERK1/2, p-MEK1/2, TrKB and BDNF in the striatal neurons were decreased. From these findings, it was concluded that reduced neurotoxicity in striatal neurons following treatment with gastrodin may be attributed to its suppressive effects on the expression of $\mathrm{p}-\mathrm{ERK} 1 / 2$, p-MEK1/2, BDNF and TrKB.

\section{Introduction}

Type 1 diabetes mellitus (DM) is the most common type of chronic metabolic disorder, and is characterized by insulin deficiency, which leads to elevated blood glucose levels and disordered protein metabolism (1). Long-term hyperglycemia can result in various complications that affect the eyes, kidneys, heart, blood vessels and nerves (2). Increasing evidence has demonstrated that hyperglycemia leads to oxidative stress in the central nervous system (CNS), and brain tissue is susceptible to oxidative damage induced by diabetes (3). It has been reported that neuronal death may be caused by diabetes-mediated neurotoxicity (4). Furthermore, insulin resistance in DM may cause $\beta$-amyloid deposition, which further exacerbates neurotoxicity, causing damage to the brain and affecting cognitive function (5). Previous studies have indicated that hyperglycemia is associated with neurotoxicity 
and neurotrophic alterations $(6,7)$, although the underling molecular mechanism remains to be fully elucidated.

Regarding neurotrophic factors, brain-derived neurotrophic factor (BDNF) is a member of the neurotrophin family, which is widely expressed in the CNS. BDNF functions through binding to its receptor, tyrosine receptor kinase $\mathrm{B}$ (TrKB). Through its regulation of Trk receptors, BDNF affects the assembly of the cytoskeleton, the pattern of innervation, synaptic strength, plasticity and the expression of functionally important proteins (8-10). Although numerous studies have reported that $\mathrm{BDNF}$ and mitogen-activated protein kinase (MAPK) signaling pathways enhance $\mathrm{Ca}^{2+}$-dependent excitotoxicity, its specific mechanism remains to be fully elucidated. In a number of neurological diseases, glutamate has been reported to accumulate in the extracellular space. The excessive synaptic release of glutamate and activation of glutamate receptors subsequently leads to the dysregulation of $\mathrm{Ca}^{2+}$ homeostasis and the induction of excitotoxic cell death (11). In addition, increased extracellular concentrations of glutamate or its analogs may activate MAPK signaling pathways in primary neuronal cultures (12). In a separate study, it was suggested that BDNF increases glutamate release and N-methyl-D-aspartate (NMDA) channel-gated $\mathrm{Ca}^{2+}$ influx via TrkB, and thus regulates the frequency and amplitude of $\mathrm{Ca}^{2+}$ oscillations (13). A study on diabetic rats reported the downregulation of glutamate decarboxylase, subsequently leading to the accumulation of glutamate (14). Impairment of glutamate transporters is involved in various neurological diseases, including Alzheimer's disease (15). A previous study on DM revealed increased functional activity and augmented sensitivity to the regulation of glutamate receptors, NMDA and $\alpha$-amino-3-hydroxy-5-methyl-4-isoxa zolepropionate (AMPA) (14). These factors may contribute to glutamate-mediated excitotoxicity in DM, as examined in the present study.

In chronic diseases, the release of glutamate from presynaptic membranes induces the influx of large quantities of calcium through NMDA and AMPA receptors, subsequently activating MAPK signaling pathways and BDNF. The extracellular signal-regulated kinase (ERK) signaling pathway is a major branch of the MAPK pathway. MAPK resides in the upstream region of the ERK pathway and induces ERK1/2 by phosphorylating its threonine and tyrosine residues (16). ERK signaling is known to be essential in regulating cellular proliferation, differentiation and survival, due to its effect on translational and transcriptional events $(17,18)$. It has also been reported that the ERK signaling pathway has a neuroprotective effect in the response of hippocampal neurons to glutamate-induced excitotoxicity (19).

The striatum constitutes the largest component of the basal ganglia, which integrates signals from the cerebral cortex and is central to the appropriate selection of behavioral action. As a key subcortical region, the role of the striatum has been investigated in classical motor disorders, including Parkinson's disease, Huntington's disease and Tourette syndrome, in addition to depression, and learning and memory (20-24). Approximately $95 \%$ of the striatal neurons are inhibitory projection neurons, termed medium spiny neurons (MSNs) characterized by their high spine density and GABAergic-inhibitory outputs $(25,26)$. Neurotoxicity and neurodegeneration induced by dopamine, glutamate and environmental conditions, such as overexposure to manganese, frequently occur in numerous neurological conditions, and the loss of MSNs is a prominent feature $(27,28)$.

Gastrodin is the primary chemical constituent of Gastrodia elata Blume, a traditional Chinese medicine. In view of the primary therapeutic effects of gastrodin in the CNS, the pharmacokinetics of the herbal agent in the brain has attracted increasing attention. Gastrodin, a phenolic glycoside chemically known as 4-hydroxybenzyl alcohol-4-O- $\beta$-D-glucopyr anoside, is considered to be the main bioactive constituent of Rhizoma gastrodiae. With the molecular formula, $\mathrm{C}_{13} \mathrm{H}_{18} \mathrm{O}_{7}$, and a molecular weight of $286 \mathrm{Da}$, gastrodin readily dissolves in methanol, ethanol and water, but not in chloroform or ether (29). Gastrodin is able to pass though the blood-brain barrier and readily gains access to brain tissue following its entry into the systemic circulation. Lin et al (30) reported that gastrodin was detectable in the brain 5 min after intravenous administration $(50 \mathrm{mg} / \mathrm{kg})$, reaching a peak brain concentration after $15 \mathrm{~min}$, in rats. It is well-documented that gastrodin exhibits analgesic, sedative, hypnagogic, anticonvulsant, antiepileptic and antineurodegenerative properties (31). In addition, gastrodin affects apoptosis and glutamate-induced intracellular $\mathrm{Ca}^{2+}$ increases, indicating that the $\mathrm{Ca}^{2+}$ channel is a promising target of gastrodin. Gastrodin may exert its neuroprotective effects by inhibiting excitotoxicity (32-34); however, the specific molecular mechanism underlying the association between gastrodin and neurotoxicity remains to be fully elucidated.

In light of the aforementioned studies, the present study aimed to investigate the association between BDNF, TrKB, phosphorylated (p-)ERK1/2, p-MAPK kinase (MEK)1/2, excitotoxicity, glutamate release and the effects of early intervention with gastrodin in the striatum in DM. It is anticipated that the results of the present study may provide a biochemical and molecular basis for the neuroprotective effects of gastrodin in DM-induced excitotoxicity in striatal neurons.

\section{Materials and methods}

Animals and induction of diabetes. All institutional and national guidelines for the care and use of laboratory animals were followed. A total of 70 male Sprague-Dawley rats (age, 9 weeks; weight, 250-300 g) were purchased from Chendu Dossy Experimental Animals Co., Ltd (Chendu, China) and were provided with a standard rodent diet and water ad libitum; rats were maintained at $23 \pm 1^{\circ} \mathrm{C}$ in a specific-pathogen-free environment with a 12-hour light/dark cycle and $60 \pm 10 \%$ relative humidity. Following adaptation for 2 weeks, type 1 DM was induced using a single intraperitoneal injection of streptozotocin (STZ) at $65 \mathrm{mg} / \mathrm{kg}$. Rats with blood glucose levels $>16.7 \mathrm{mmol} / 1$ for 2 consecutive days were considered to be diabetic. In the present study, the rats were randomly divided into four groups: i) NC9W group, normal control rats gavaged with normal saline $(0.4 \mathrm{ml} / 100 \mathrm{~g} / \mathrm{day})$ and fed for 6 weeks; ii) DM9W + S group, STZ-induced DM rats gavaged with normal saline $(0.4 \mathrm{ml} / 100 \mathrm{~g} /$ day $)$ for 6 weeks at 3 weeks post-diabetes induction; iii) DM9W + G60 group, STZ-induced $\mathrm{DM}$ rats gavaged with gastrodin $(60 \mathrm{mg} / \mathrm{kg} / \mathrm{day}$, dissolved in saline, $0.4 \mathrm{ml} / 100 \mathrm{~g} /$ day) for 6 weeks at 3 weeks post-diabetes 
induction; and iv) DM9W + G120 group, STZ-induced DM rats gavaged with gastrodin $(120 \mathrm{mg} / \mathrm{kg} / \mathrm{day}$, dissolved in saline, $0.4 \mathrm{ml} / 100 \mathrm{~g} / \mathrm{day}$ ) for 6 weeks at 3 weeks post-diabetes induction.

Western blotting. The striatal tissue was freshly removed from the brain of each rat and homogenized with radioimmunoprecipitation assay buffer [cat. no. 9806; Cell Signaling Technology (CST), Inc., Danvers, MA, USA] containing a 10X protease inhibitor cocktail (1:100; cat. no. 5871; CST, Inc.) and 10X phosphatase inhibitor cocktail (1:100; cat. no. 5870; CST, Inc.). Following complete lysis of the tissue, the homogenates were centrifuged at $3,028 \mathrm{x}$ g for $10 \mathrm{~min}$ at $4^{\circ} \mathrm{C}$. The supernatants were collected, and the protein concentration was determined using a bicinchoninic acid protein assay kit. Equal quantities of sample protein $(30 \mu \mathrm{g})$ were separated on $10 \%$ SDS-PAGE gels and transferred onto a polyvinylidene difluoride membrane (EMD Millipore, Billerica, MA, USA). Following blocking with 5\% skim milk for $2 \mathrm{~h}$ at room temperature, the membranes were incubated at $4^{\circ} \mathrm{C}$ overnight with the following primary antibodies: Rabbit p-MEK1/2 monoclonal (1:1,000; cat. no. 9154s; CST, Inc.), rabbit p-ERK1/2 monoclonal $(1: 1,000$; cat. no. 4370s; CST, Inc.), mouse TrKB monoclonal (1:2,000; cat. no. sc-377218; Santa Cruz Biotechnology, Inc., Dallas, TX, USA), mouse BDNF monoclonal (1:2,000; cat. no. ab203573; Abcam, Cambridge, UK), rabbit MEK1/2 monoclonal (1:1,000; cat. no. 8727s; CST, Inc.), rabbit ERK1/2 monoclonal (1:1,000; cat. no. 4695s; CST, Inc.), and rabbit $\beta$-tubulin monoclonal $(1: 1,000$; cat. no. 15115; CST, Inc.). Following incubation for $2 \mathrm{~h}$ at room temperature with goat-anti-rabbit $(1: 1,000$; cat. no. 31460; Thermo Fisher Scientific, Inc., Waltham, MA, USA) and goat-anti-mouse (1:1,000; cat. no. 31430; Thermo Fisher Scientific, Inc.) immunoglobulin G H\&L secondary antibodies, the blots were developed with enhanced chemiluminescence, and densitometric analysis of the film was performed using ImageJ software (version 1.4.3.67; National Institutes of Health, Bethesda, MD, USA).

Immunofluorescence. At designated time points, the rats were anesthetized with ketamine $(75 \mathrm{mg} / \mathrm{kg})$ and xylazine $(10 \mathrm{mg} / \mathrm{kg})$, administered intraperitoneally, and then perfused transcardially with saline, followed by $4 \%$ paraformaldehyde. Following perfusion, the brain was removed, fixed in $4 \%$ formaldehyde for $48 \mathrm{~h}$ at room temperature, dehydrated in an ascending ethanol series, cleared with xylene and embedded in paraffin blocks. The $3 \mu \mathrm{m}$ thick paraffin sections were deparaffinized and hydrated through a descending series of gradient ethanol. The tissues were incubated in citrate buffer for antigen retrieval. Subsequently, the tissue sections were incubated with 5\% normal goat serum (Beijing Biosynthesis Biotechnology Co., Ltd, Beijing, China) for $2 \mathrm{~h}$ at room temperature and then overnight at $4^{\circ} \mathrm{C}$ with the following primary antibodies: Mouse dopamine- and cAMP-regulated neuronal phosphoprotein (DARPP)-32 monoclonal (1:100; cat. no. sc-271111; Santa Cruz Biotechnology, Inc.), rabbit p-ERK1/2 monoclonal (1:1,000; cat. no. 4370s; Cell CST, Inc.), rabbit MEK1/2 monoclonal (1:1,000; cat. no. 8727s; CST, Inc.) and rabbit ERK1/2 monoclonal (1:1,000; cat. no. 4695s; CST, Inc.). This was followed by incubation with goat-anti-rabbit immunoglobulin G H\&L Cross-Adsorbed Alexa Fluor 488 (1:100; cat. no. A11008; Thermo Fisher Scientific, Inc.) or goat-anti-mouse immunoglobulin G H\&L Cross-Adsorbed Alexa Fluor 546 (1:100; cat. no. A11003; Invitrogen; Thermo Fisher Scientific, Inc.) secondary antibodies for $2 \mathrm{~h}$ at room temperature. Finally, the tissue sections were washed with PBS. Images were captured at magnification, x600 and x1,000 under a confocal fluorescence microscope (FV1000; Olympus Corporation, Tokyo, Japan;) with ChemiDoc XRS+ (Bio-Rad Laboratories, Inc., Hercules, CA, USA).

Hematoxylin and eosin $(H \& E)$ staining. The aforementioned tissue sections were were first incubated with hematoxylin for 5 min and subsequently washed with $1 \%$ ethanol hydrochloride for $3 \mathrm{sec}$ at room temperature. Following washing with water, the sections were stained with eosin. To assess the abnormalities in neurons, the sections were examined at $x 400$ magnification under a light microscope in a blinded manner.

Amino acid analysis. The extracellular levels of glutamate were measured using microdialysis followed by high-pressure-liquid-chromatography (HPLC) in the striatum. HPLC uses a liquid as the mobile phase and a high-pressure infusion system to pump a single solvent with different polarities or a mixed solvent of different ratios, into a column packed with a stationary phase; the components in the column are subsequently separated. The derivative reagent was prepared by dissolving $0.05 \mathrm{mg}$ o-phthalaldehyde in $1 \mathrm{ml}$ methanol to which $500 \mu 1 \beta$-mercaptoethanol and $0.1 \mathrm{~mol} / \mathrm{l}$ Na-tetraborate was added to make $10 \mathrm{ml}$. An HPLC device (Agilent 1200; Agilent Technologies, Inc., Santa Clara, CA, USA) with two mobile phases was used. Mobile phase A consisted of $0.25 \%$ (v\%) tetrahydrofuran in $0.03 \mathrm{~mol} / \mathrm{l}$ sodium acetate solution (solution $\mathrm{pH}, 7.2$ ). Mobile phase B consisted of $80 \%$ (v\%) acetonitrile and $20 \%$ (v\%) $0.01 \mathrm{~mol} / 1$ sodium acetate solution. Glutamate standards were weighed and prepared into amino acid standard solutions at different concentrations (1.00, 2.50, $5.00,10.00,25.00,50.00$ and $100.00 \mathrm{mg} / \mathrm{l})$. A $20-\mu 1$ sample solution at each concentration was detected to plot a standard curve with the concentrations represented on the $\mathrm{X}$ axis and the peak area serving as the $\mathrm{Y}$ axis. The optimal fit for all standards was 0.9992 . Glutamate concentrations in the striatum were measured in each group based on this analytical method.

Statistical analysis. Data are expressed as the mean \pm standard deviation and were analyzed with SPSS 17.0 statistical software (SPSS, Inc., Chicago, IL, USA). Comparisons among groups were performed using one-way analysis of variance and pairwise comparison was performed using the least significant difference t-test. $\mathrm{P}<0.05$ was considered to indicate a statistically significant difference.

\section{Results}

Early intervention with gastrodin ameliorates neuronal injury in the striatum. The neurons of the striatum were examined by H\&E staining (Fig. 1). In the normal control (NC9W) group, the striatal neurons were arranged in an orderly manner. The neurons were well-defined, exhibiting a conspicuous round or oval nucleus with discrete chromatin formations. By contrast, 

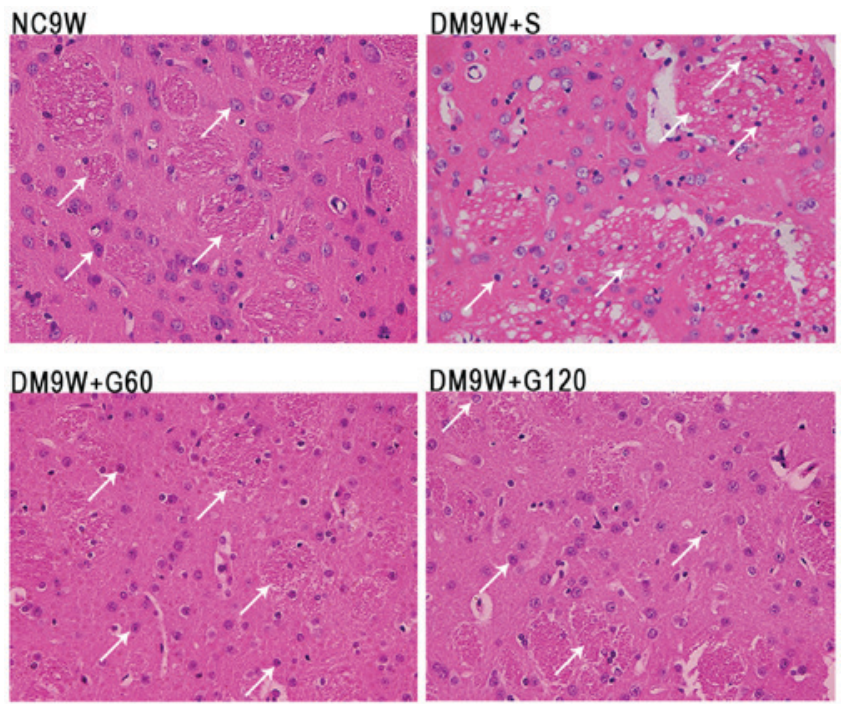

Figure 1. Effect of gastrodin on the histopathological alterations in type 1 DM rat models. Histopathological alterations were evaluated by hematoxylin-eosin staining. Representative images of the NC9W, DM9W + S, DM9W + G60 and DM9W + G120 groups are shown (magnification, $\mathrm{x} 400$ ). The nuclei of the affected neurons (white arrows) were condensed in the DM9W + S group compared with those in the NC9W group; the neuronal nuclei (white arrows) in the striatum of the DM9W + G60 and DM9W + G120 groups were comparable with those in the DM9W + S group NC, normal control; DM, diabetic mellitus; S, normal saline; G60, gastrodin (60 mg/kg/day); Gl20, gastrodin (120 mg/kg/day).

the striatal neurons in DM9W $+\mathrm{S}$ were arranged in a disorderly manner with a pyknotic or shrunken nucleus. Furthermore, the striatal tissue appeared vacuolated. Compared with the NC9W group, neuronal damage was less evident in the DM9W + G60 and DM9W + G120 groups.

Early intervention with gastrodin decreases the expression of $p$-ERK1/2 in MSNs. MEK1/2, ERK1/2 and p-ERK1/2 (red, Alexa Fluor 546) immunofluorescence was primarily localized to the MSNs (green, Alexa 488) in the striatum. On closer scrutiny, ERK1/2, MEK1/2 and p-ERK1/2 immunofluorescence was detected in the cytoplasm of the MSNs; occasionally, the MSNs exhibited the ERK1/2, MEK1/2 and p-ERK1/2 in the nucleus (Fig. 2A-D). Double immunofluorescence labeling of $\mathrm{p}$-ERK1/2 revealed significantly increased expression levels in the MSNs of the DM9W $+\mathrm{S}$ group compared with that of the NC9W group $(\mathrm{P}<0.001)$. However, expression of p-ERK1/2 in the MSNs of the DM9W + G60 and DM9W + G120 groups was significantly attenuated compared with that in the DM9W $+\mathrm{S}$ group $(\mathrm{P}<0.01 ;$ Fig. $2 \mathrm{~B})$.

Gastrodin decreases the phosphorylation of $M E K 1 / 2$ and ERK1/2 and the expression of BDNF and TrKB. To examine the contribution of MEK1/2, ERK1/2, BDNF and TrKB to the neuroprotective effect of gastrodin, the expression levels of these biomarkers were determined by western blotting (Fig. 3). The results demonstrated that the expression of $\mathrm{p}-\mathrm{MEK} 1 / 2$ was significantly increased in the DM9W $+\mathrm{S}$ group compared with that in the NC9W group $(\mathrm{P}<0.001)$. In the DM9W + G60 group, the expression of $\mathrm{p}-\mathrm{MEK} 1 / 2$ was significantly lower compared with that in the DM9W $+\mathrm{S}$ group $(\mathrm{P}<0.001)$; and the expression of $\mathrm{p}-\mathrm{MEK} 1 / 2$ in the DM9W + G120 group was significantly higher compared with that in the GM9W + G60 group $(\mathrm{P}<0.001$; Fig. 3Aa-c). Additionally, the expression of p-ERK1/2 was significantly higher in the DM9W $+\mathrm{S}$ group compared with that in the NC9W group $(\mathrm{P}<0.001)$, and was significantly lower in the DM9W + G60 group when compared with that in the DM9W $+\mathrm{S}$ group $(\mathrm{P}<0.001$; Fig. 3Ba-c). Alterations in the expression levels of BDNF and TrKB followed the same trend as those for p-ERK1/2 (Fig. 3Ca-c).

Gastrodin decreases glutamate concentration. The extracellular levels of glutamate were measured by HPLC. Compared with the NC9W group, glutamate levels were significantly increased in the DM9W $+\mathrm{S}(\mathrm{P}<0.001)$ and $\mathrm{DM} 9 \mathrm{~W}+\mathrm{G} 120$ $(\mathrm{P}<0.001)$ groups. However, the glutamate level in the DM9W + G60 group was significantly lower compared with that in the DM9W $+\mathrm{S}$ group $(\mathrm{P}<0.01)$; no significant differences were detected between the DM9W $+\mathrm{S}$ and DM9W + G120 groups (Fig. 4).

\section{Discussion}

Previous studies have reported that experimentally-induced diabetes may reduce the proliferation of granular cells and enhance neuronal death (necrosis/apoptosis) in the CA3 and dentate gyrus regions $(35,36)$. Duration-associated apoptosis is likely to account for the loss of neurons and the concomitant emergence of cognitive impairments observed in diabetic animals (37). In the present study, it was demonstrated that striatal neurons underwent evident pathological alterations 9 weeks subsequent to diabetic induction in rats. Notably, these pathological alterations in the striatal neurons were ameliorated following early intervention with gastrodin.

Glutamate is the primary excitatory neurotransmitter in the mammalian brain. It has been demonstrated to be important in numerous processes in the brain, including neurodevelopmental processes, such as differentiation, migration and survival. By contrast, glutamate has also been implicated in acute neurodegeneration, chronic neurodegeneration, the stress response and anxiety disorders. Furthermore, the ERK/MAPK signaling cascade is essential in neuronal cell survival and death, generally depending on its magnitude and duration of activation. However, evidence suggests that the activation of ERK1/2 also contributes to cell death in certain cell types and organs under specific conditions (38). In addition, the excessive release of glutamate and the subsequent influx of calcium are associated with a number of neurological insults that result in neuronal death (39). Glutamate may lead to the persistent activation of ERK1/2, which is associated with neuronal cell death (40). This is due to the fact that the stimulation of glutamate receptors and influx of $\mathrm{Ca}^{2+}$ are associated with excitotoxic injury, leading to the phosphorylation of p44/42 MAPK in neurons. Extracellular glutamate may be released from neurons and astrocytes, and according to other studies, activated microglia also release certain amounts of glutamate under various pathological processes, including inflammation $(15,41)$. Therefore, the possibility that glutamate may be released from glial cells and neurons in the striatal tissue in DM should be considered.

The activation of ERKs has been revealed to contribute to neuronal cell death in certain in vitro models of neurotoxicity. Persistent activation of ERK1/2 contributes to glutamate-induced 

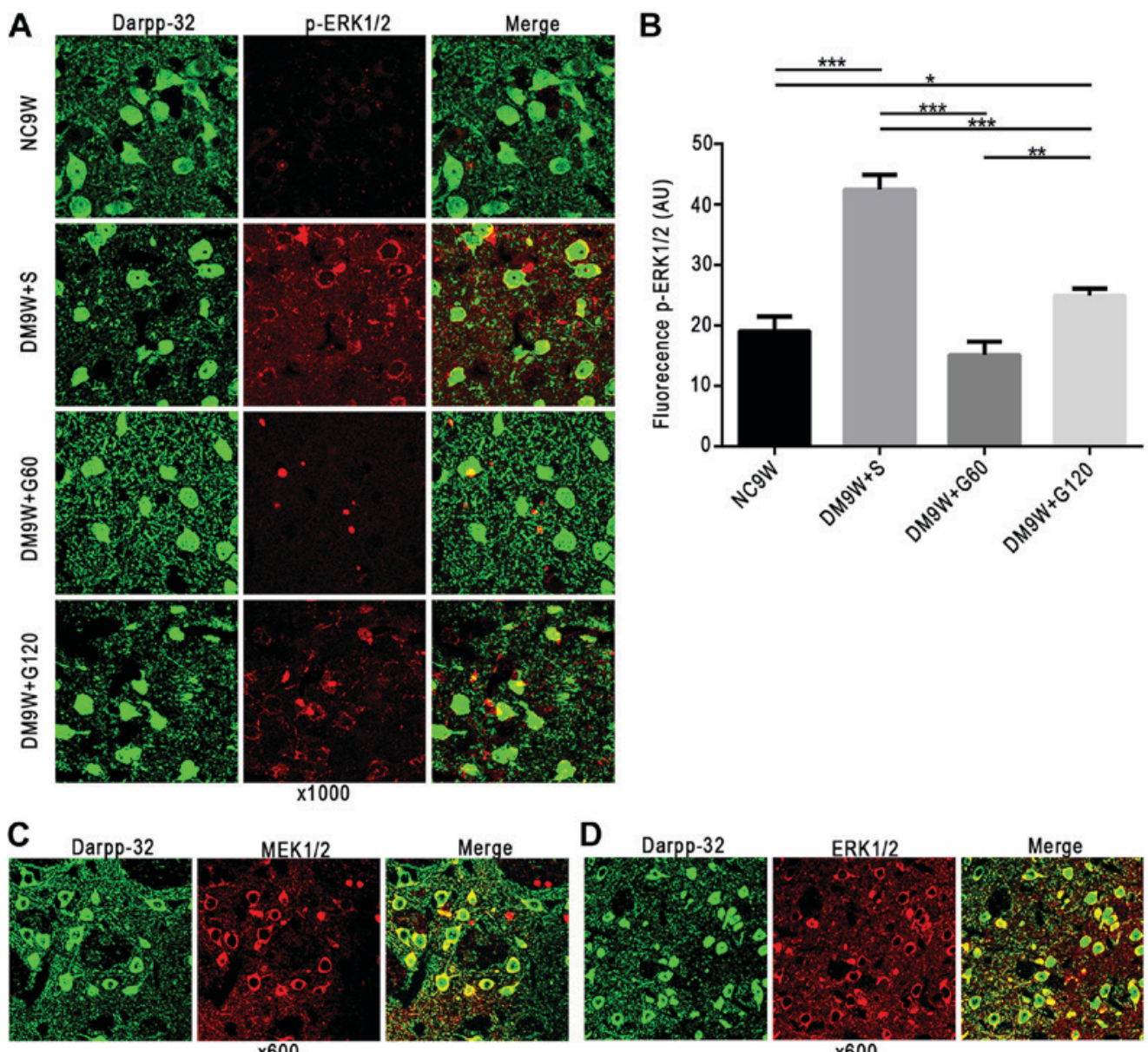

Figure 2. Localization of MEK1/2, ERK1/2 and p-ERK1/2 in MSNs of the striatum. MEK1/2, ERK1/2 and p-ERK1/2 are shown in red (Alexa Fluor 546), and MSN marker DARPP-32 in green (Alexa 488). (A) Gastrodin treatment decreased the expression levels of p-ERK1/2 in MSNs; p-ERK1/2 was localized preferentially in the cytoplasm. (B) Quantification of fluorescence intensity is presented as the mean \pm standard deviation. ${ }^{*} \mathrm{P}<0.05,{ }^{* * *} \mathrm{P}<0.01$ and ${ }^{* * * *} \mathrm{P}<0.001$. (C) MEK1/2 and (D) ERK1/2 were also localized preferentially in the cytoplasm. DARPP-23, dopamine- and cAMP-regulated neuronal phosphoprotein-32; DM, diabetes mellitus; ERK, extracellular signal regulated kinase; G60, gastrodin (60 mg/kg/day); Gl20, gastrodin (120 mg/kg/day); MEK, mitogen-activated protein kinase kinase; MSNs, medium spiny neurons; NC, normal control; S, normal saline; p-, phosphorylated.

oxidative toxicity (42-45), which is consistent with the results of the present study. ERK also contributes to cell death through the suppression of anti-apoptotic signaling molecule RAC- $\alpha$ serine/threonine-protein kinase (34). The results of the present study indicated that, 9 weeks following the induction of diabetes, the expression levels of p-ERK1/2 and glutamate were significantly elevated. Notably, following early intervention with gastrodin, glutamate levels and the expression of ERK1/2 were reduced. As a corollary, it may be that gastrodin reduces glutamate-induced excitotoxicity by reducing the content of glutamate and suppressing the expression level of ERK.

It is widely known that BDNF has a neuroprotective effect by preventing the neuronal death induced by metabolic and oxidative stress and excitotoxicity, and modulating calcium responses to NMDA and AMPA receptors, which may be associated with the MAPK signaling pathway. Its specific mechanism remains to be fully elucidated, but may involve the enhancement of antioxidant systems (46-48). However, certain neurotrophins may have opposing effects on different types of cell death within the same neuron $(49,50)$. Previous reports have indicated that certain trophic factors have the capacity to exacerbate excitotoxicity $(13,51)$ which is consistent with the results of the present study on diabetes; however, the specific mechanisms involved remain to be elucidated. Previous studies have reported that excitotoxicity caused by glutamate and the deregulation of intracellular $\mathrm{Ca}^{2+}$ homeostasis may contribute to numerous forms of pathological neuronal death, including acute injuries, such as hypoxia, hypoglycemia and seizures, and chronic neurodegenerative disorders, such as Parkinson's, Alzheimer's and Huntington's disease (51). Furthermore, it has been reported that glutamate-induced intracellular $\mathrm{Ca}^{2+}$ accumulation is time- and dose-dependent (52). Previous studies have demonstrated that AMPA and NMDA receptors are involved in the regulation of BDNF (41). The upregulation of AMPA and NMDA receptor subunits, mediated by the binding of BDNF to Trk receptors, enhances calcium responses to NMDA, increasing neuronal vulnerability to excitotoxic necrosis (51).

The results of the present study demonstrated marked upregulation in the levels of glutamate, BDNF and TrKB in the DM rats, and this may enhance neurotoxicity resulting in neuronal injury. These results suggested that gastrodin was able to decrease neuronal injury by reducing glutamate, and the overexpressed BDNF and TrKB. Therefore, damage to striatal neurons, as observed in the present DM rat model, may be attributed to 

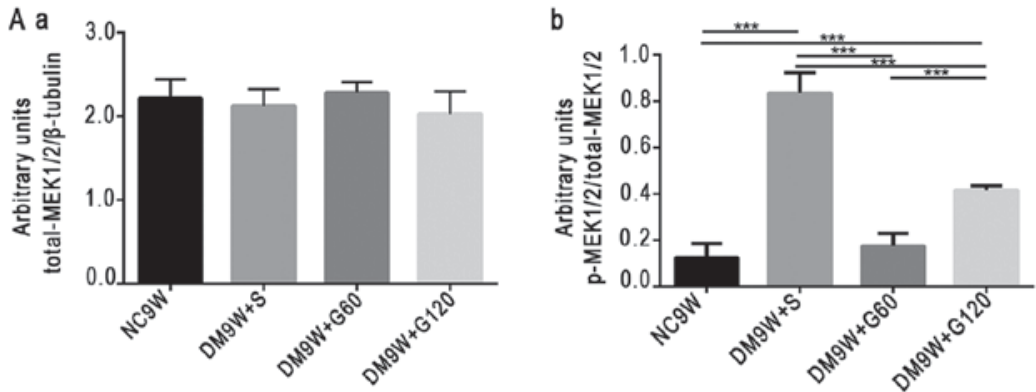

C

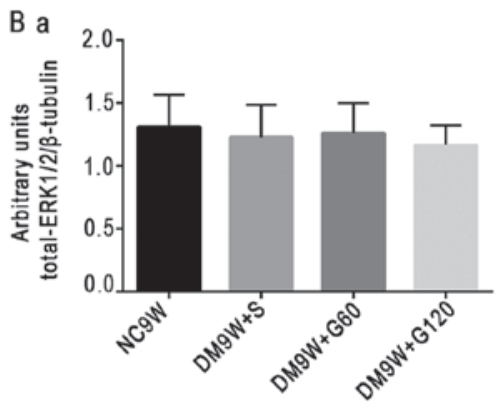

b
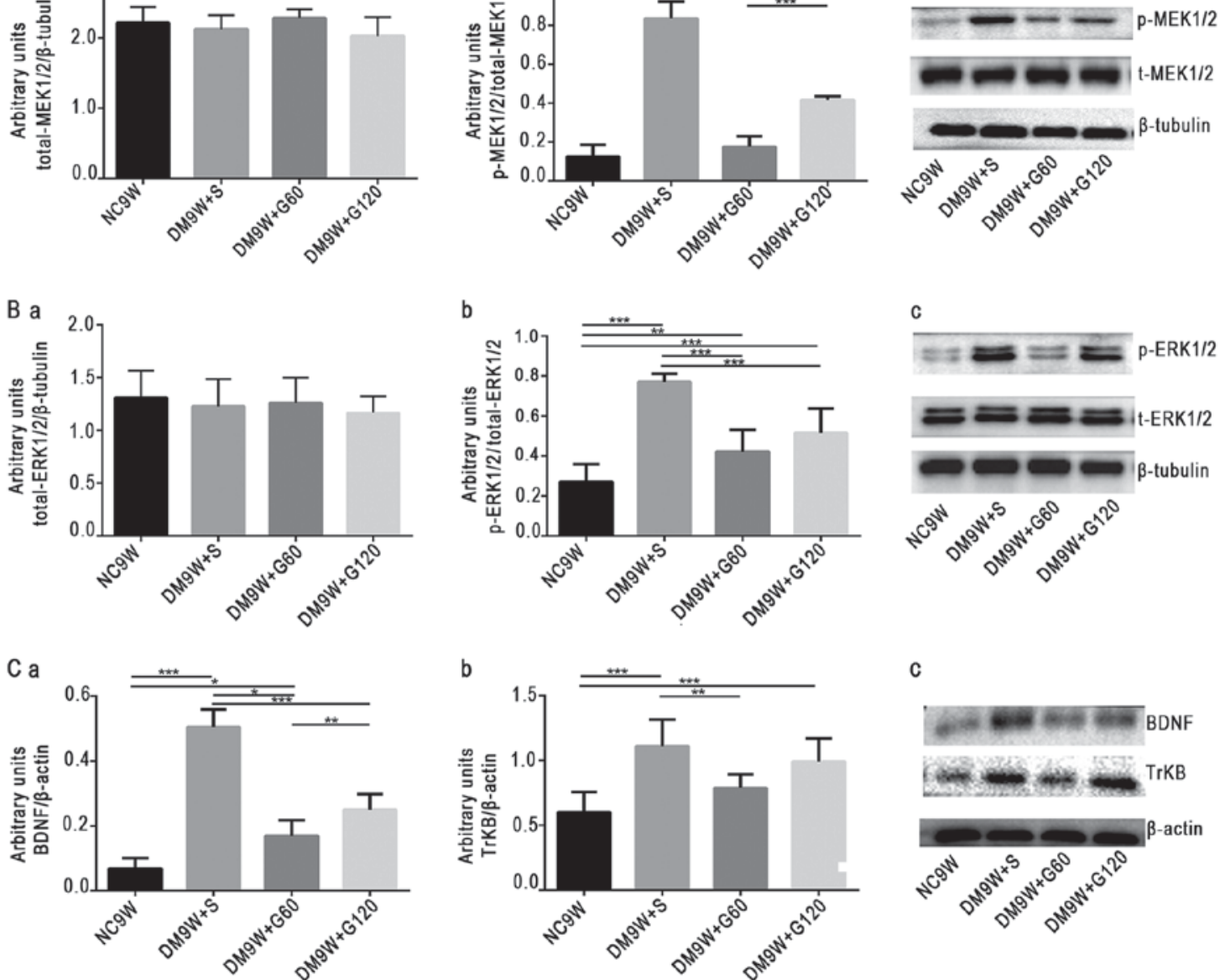

Figure 3. Western blot analysis of protein expression levels of p-ERK1/2, p-MEK1/2, BDNF and TrKB. Gastrodin decreased the expression levels of p-ERK1/2, p-MEK1/2, BDNF and TrKB in the striatum of type 1 DM rats. (A) Densitometric quantification of (a) t-MEK1/2 and (b) p-MEK1/2:t-MEK1/2 from (c) western blots. (B) Densitometric quantification of (a) t-ERK1/2 and (b) p-ERK1/2:t-ERK1/2 from (c) western blots. (C) Densitometric quantification of (a) BDNF: $\beta$-actin and (b) TrKB: $\beta$-actin from (c) western blots. The bars represent total gray values (mean \pm standard deviation). ${ }^{*} \mathrm{P}<0.05,{ }^{* *} \mathrm{P}<0.01$ and ${ }^{* * * *} \mathrm{P}<0.001$. BDNF, brain-derived neurotrophic factor; DM, diabetes mellitus; ERK, extracellular signal regulated kinase; G60, gastrodin (60 mg/kg/day); Gl20, gastrodin (120 mg/kg/day); MEK, mitogen-activated protein kinase kinase; NC, normal control; p-, phosphorylated; S, normal saline; t-, total; TrKB, tyrosine receptor kinase B.

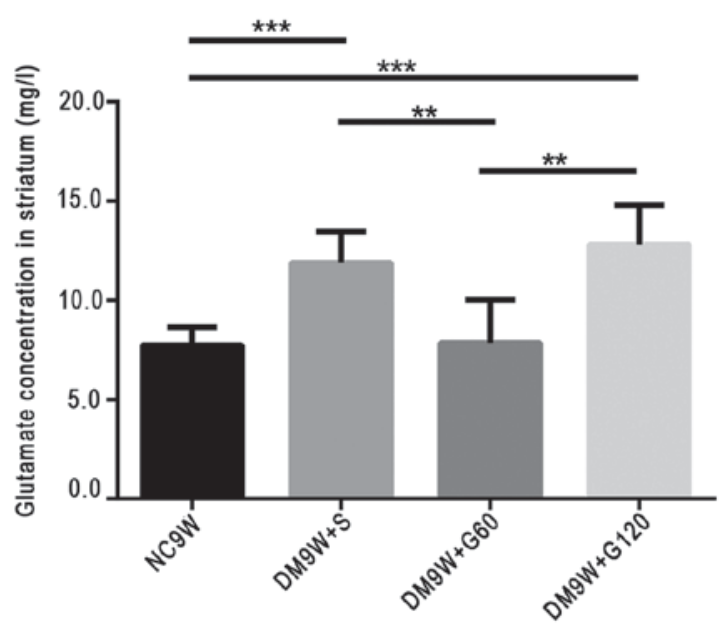

Figure 4. High-pressure-liquid-chromatography determination of the extracellular release of glutamate in diabetic rats and in diabetic rats treated with gastrodin intervention. Gastrodin decreased the content of glutamate in the striatum of the type 1 diabetic rats. Bars represent glutamate concentration (mean \pm standard deviation). ${ }^{* *} \mathrm{P}<0.01$ and ${ }^{* * *} \mathrm{P}<0.001$. DM, diabetes mellitus; G60, gastrodin (60 mg/kg/day); Gl20, gastrodin (120 mg/kg/day); $\mathrm{NC}$, normal control; S, normal saline. excitotoxicity. An increase in glutamate in striatal tissue may be the primary contributing factor to neuronal damage. Notably, an increase in extracellular glutamate levels was coupled with the overactivation of p-ERK1/2, p-MEK1/2, BDNF and TrKB. It is possible that these factors and signaling pathways collectively lead to neuronal dysfunction and structural alterations (Fig. 5). Furthermore, the results revealed that gastrodin protected neurons through inhibiting the activation of these factors and thus reducing the levels of neurotoxicity in experimentally-induced diabetes. Gastrodin has been described as a 'top grade medicine', capable of improving health and extending life without toxicity, and may be used long term without causing harm (29). A previous study showed that pretreatment with gastrodin at 60 and $120 \mathrm{mg} / \mathrm{kg}$ had neuroprotective effects (53), which is consistent with the results of the present study. Of note, in the present experimentally-induced DM model, gastrodin at a lower dose $(60 \mathrm{mg} / \mathrm{kg})$ was demonstrated to be more potent in reducing neurotoxicity levels compared with a higher dose $(120 \mathrm{mg} / \mathrm{kg})$. This suggested that gastrodin at $60 \mathrm{mg} / \mathrm{kg}$ is the optimal dose for the attenuation of diabetes-induced neurotoxicity in striatal neurons. 


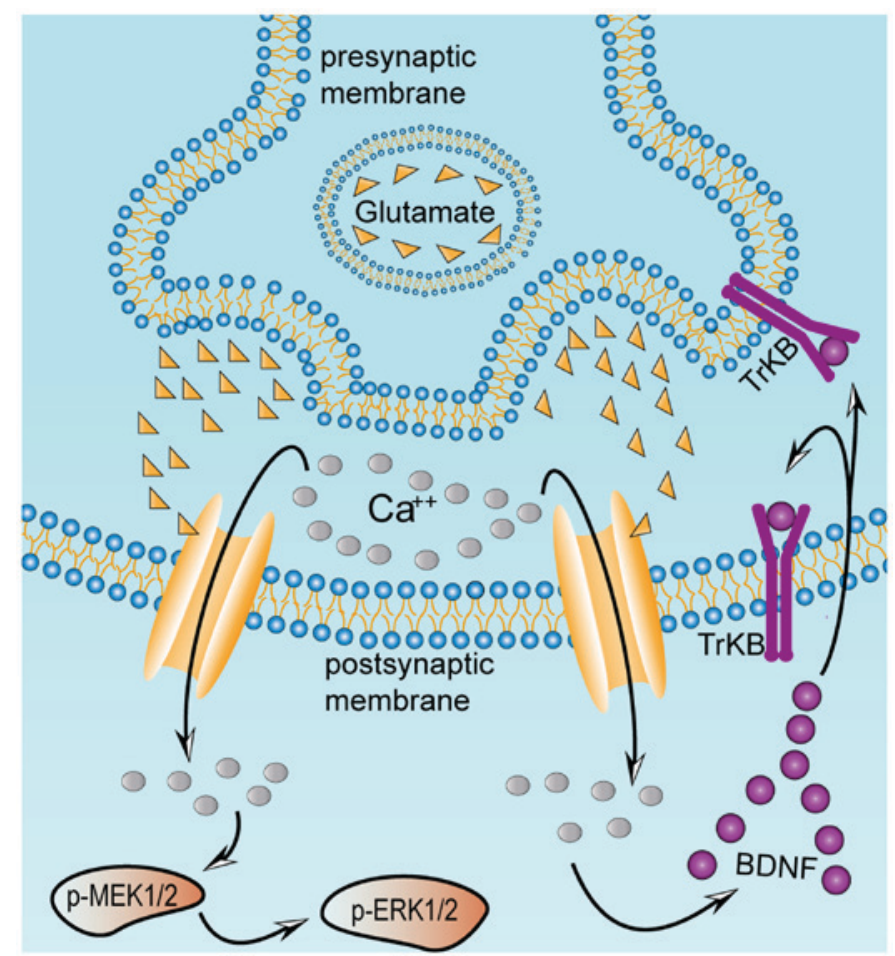

Figure 5. Excessive release of glutamate from presynaptic terminals activates BDNF, TrkB, p-MEK1/2 and p-ERK1/2 in postsynaptic neurons. Excitatory damage may be caused by chronic metabolic diseases, including diabetes mellitus. This may stimulate excessive release of glutamate, which induces a massive influx of calcium mediated by NMDA and $\alpha$-amino-3-hydroxy-5-methyl-4-isoxazolepropionate receptors in the postsynaptic striatal neurons, thereby activating the MAPK pathway and BDNF. Once BDNF secretion is activated, it binds to its receptor TrkB, localized on the presynaptic and postsynaptic membranes. Presynaptic TrkB receptors are known to mediate the enhancement of glutamate release, whereas postsynaptic TrkB receptors enhance the response of NMDA receptors to calcium ions. Excessive influx of calcium ions into the postsynaptic striatal neurons results in continuous activation of the MAPK and BDNF pathways, which ultimately causes excitatory damage to the striatum. BDNF, brain-derived neurotrophic factor; ERK, extracellular signal regulated kinase; MEK, mitogen-activated protein kinase kinase; NMDA, N-methyl-D-aspartate; p-, phosphorylated; TrKB, tyrosine receptor kinase B.

\section{Acknowledgements}

Not applicable.

\section{Funding}

The present study was supported in part by The National Natural Sciences Foundation of China (grant nos. 81760149, 81460210, 81360176, 31760292 and 81200840), The National University Students Innovation and Entrepreneurship Training Program (grant no. 201710678004), The Department of Science and Technology of Yunnan Province (grant no. 2013HB078), The Joint Special Funds for the Department of Science and Technology of Yunnan Province-Kunming Medical University [grant nos. 2017FE468 (-171), 2017FE468 and 2014FZ007] and The Yunnan provincial Education Commission (grant nos. 2018JS156 and 2018Y040).

\section{Availability of data and materials}

All data generated or analyzed during this study are included in this published article.

\section{Authors' contributions}

YYZ and DL designed the project, and contributed to the analysis of data and finalization of the manuscript. YHQ and RZ performed the majority of the experiments, participated in discussion and analysis of data, and prepared the first draft of the manuscript. QW and QL conducted part of experiments, and participated in discussion and analysis of data. YDL designed and guided the use of gastrodin. ZYQ and ZHY performed paraffin embedding, sectioning and H\&E staining. ZHM guided the molecular biology experiment and analysis of data. XJL, MYZ, XW and XYL helped with removal of tissue samples and took care of the experimental rats. QW revised the manuscript.

\section{Ethics approval and consent to participate}

This study was approved by the Medical Ethics Committee of Kunming Medical University. All institutional and national guidelines for the care and use of laboratory animals were followed.

\section{Patients consent for publication}

Not applicable.

\section{Competing interests}

All authors declare that they have no competing interests.

\section{References}

1. Ding Y, Sun X and Shan PF: MicroRNAs and cardiovascular disease in diabetes mellitus. Biomed Res Int 2017: 4080364, 2017. 
2. Gispen WH and Biessels GJ: Cognition and synaptic plasticity in diabetes mellitus. Trends Neurosci 23: 542-549, 2000.

3. Ashafaq M, Varshney L, Khan MH, Salman M, Naseem M, Wajid S and Parvez S: Neuromodulatory effects of hesperidin in mitigating oxidative stress in streptozotocin induced diabetes. Biomed Res Int 2014: 249031, 2014.

4. Kong FJ, Ma LL, Guo JJ, Xu LH, Li Y and Qu S: Endoplasmic reticulum stress/autophagy pathway is involved in diabetes-induced neuronal apoptosis and cognitive decline in mice. Clin Sci (Lond) 132: 111-125, 2017.

5. Zhang Y, Huang NQ, Yan F, Jin H, Zhou SY, Shi JS and Jin F: Diabetes mellitus and Alzheimer's disease: GSK-3 $\beta$ as a potential link. Behav Brain Res 339: 57-65, 2018.

6. Li Y, Zhang Y, Wang L, Wang P, Xue Y, Li X, Qiao X, Zhang X, $\mathrm{Xu} \mathrm{T}$, Liu G, et al: Autophagy impairment mediated by S-nitrosation of ATG4B leads to neurotoxicity in response to hyperglycemia. Autophagy 13: 1145-1160, 2017.

7. Zhong Y, Zhu Y, He T, Li W, Li Q and Miao Y: Brain-derived neurotrophic factor inhibits hyperglycemia-induced apoptosis and downregulation of synaptic plasticity-related proteins in hippocampal neurons via the PI3K/Akt pathway. Int J Mol Med 43: 294-304, 2019.

8. Huang EJ and Reichardt LF: Trk receptors: Roles in neuronal signal transduction. Annu Rev Biochem 72: 609-642, 2003.

9. Yamada $K$ and Nabeshima T: Brain-derived neurotrophic factor/TrkB signaling in memory processes. J Pharmacol Sci 91: 267-270, 2003

10. Huang EJ and Reichardt LF: Neurotrophins: Roles in neuronal development and function. Annu Rev Neurosci 24: 677-736, 2001.

11. Arundine $\mathbf{M}$ and Tymianski M: Molecular mechanisms of calcium-dependent neurodegeneration in excitotoxicity. Cell Calcium 34: 325-337, 2003

12. Rivera-Cervantes MC, Castañeda-Arellano R, Castro-Torres RD Gudino-Cabrera G, Feria y Velasco AI, Camins A and Beas-Zarate C: P38 MAPK inhibition protects against glutamate neurotoxicity and modifies NMDA and AMPA receptor subunit expression. J Mol Neurosci 55: 596-608, 2015.

13. Sakai N, Yamada M, Numakawa T, Ogura A and Hatanaka $H$ : $\mathrm{BDNF}$ potentiates spontaneous $\mathrm{Ca} 2+$ oscillations in culture hippocampal neurons. Brain Res 778: 318-328, 1997.

14. Jayanarayanan S, Smijin S, Peeyush KT, Anju TR and Paulose CS: NMDA and AMPA receptor mediated excitotoxicity in cerebral cortex of streptozotocin induced diabetic rat: Ameliorating effects of curcumin. Chem Biol Interact 201: 39-48, 2013.

15. Junpei T, Koki F, Marie M, Takeshi S, Yuko S and Kaoru S L-glutamate released from activated microglia downregulates astrocytic L-glutamate transporter expression in neuroinflammation: The 'collusion' hypothesis for increased extracellular L-glutamate concentration in neuroinflammation. J Neuroinflammation 9: 275, 2012.

16. Li ZY, Huang Y, Yang YT, Zhang D, Zhao Y, Hong J, Liu J, Wu LJ, Zhang $\mathrm{CH}, \mathrm{Wu} \mathrm{HG}$, et al: Moxibustion eases chronic inflammatory visceral pain through regulating MEK, ERK and CREB in rats. World J Gastroenterol 23: 6220-6230, 2017.

17. Birkner K, Wasser B, Loos J, Plotnikov A, Seger R, Zipp F, Witsch E and Bittner S: The role of ERK signaling in experimental autoimmune encephalomyelitis. Int J Mol Sci 18: E1990, 2017.

18. Plotnikov A, Chuderland D, Karamansha Y, Livnah O and Seger R: Nuclear extracellular signal-regulated kinase 1 and 2 translocation is mediated by casein kinase 2 and accelerated by autophosphorylation. Mol Cell Biol 31: 3515-3530, 2011.

19. Ortuño-Sahagún D, González RM, Verdaguer E, Huerta VC, Torres-Mendoza BM, Lemus L, Rivera-Cervantes MC, Camins A and Zarate CB: Glutamate excitotoxicity activates the MAPK/ERK signaling pathway and induces the survival of rat hippocampal neurons in vivo. J Mol Neurosci 52: 366-377, 2014.

20. Shepherd GM: Corticostriatal connectivity and its role in disease Nat Rev Neurosci 14: 278-291, 2013.

21. Caligiore D, Mannella F, Arbib MA and Baldassarre G: Dysfunctions of the basal ganglia-cerebellar-thalamo-cortical system produce motor tics in Tourette syndrome. PLoS Comput Biol 13: e1005395, 2017

22. Chang C, Crottaz-Herbette S and Menon V: Temporal dynamics of basal ganglia response and connectivity during verbal working memory. Neuroimage 34: 1253-1269, 2007.

23. Murty VP, DuBrow S and Davachi L: The simple act of choosing influences declarative memory. J Neurosci 35: 6255-6264, 2015.
24. van Duinkerken E, Schoonheim MM, Steenwijk MD, Klein M, Ijzerman RG, Moll AC, Heymans MW, Snoek FJ, Barkhof F and Diamant M: Ventral striatum, but not cortical volume loss, is related to cognitive dysfunction in type 1 diabetic patients with and without microangiopathy. Diabetes Care 37: 2483-2490, 2014.

25. Gerfen CR and Surmeier DJ: Modulation of striatal projection systems by dopamine. Annu Rev Neurosci 34: 441-466, 2011.

26. Reinius B, Blunder M, Brett FM, Eriksson A, Patra K, Jonsson J, Jazin $\mathrm{E}$ and Kullander K: Conditional targeting of medium spiny neurons in the striatal matrix. Front Behav Neurosci 9: 71, 2015.

27. Stansfield KH, Bichell TJ, Bowman AB and Guilarte TR: BDNF and Huntingtin protein modifications by manganese: Implications for striatal medium spiny neuron pathology in manganese neurotoxicity. J Neurochem 131: 655-666, 2014

28. Zhang J, Saur T, Duke AN, Grant SG, Platt DM, Rowlett JK, Isacson $\mathrm{O}$ and Yao WD: Motor impairments, striatal degeneration, and altered dopamine-glutamate interplay in mice lacking PSD-95. J Neurogenet 28: 98-111, 2014.

29. Liu Y, Gao J, Peng M, Meng H, Ma H, Cai P, Xu Y, Zhao Q and Si G: A review on central nervous system effects of gastrodin. Front Pharmacol 9: 24, 2018.

30. Lin LC, Chen YF, Tsai TR and Tsai TH: Analysis of brain distribution and biliary excretion of a nutrient supplement, gastrodin, in rat. Anal Chim Acta 590: 173-179, 2007.

31. Zhan HD, Zhou HY, Sui YP, Du XL, Wang WH, Dai L, Sui F, Huo HR and Jiang TL: The rhizome of Gastrodia elata Blume-an ethnopharmacological review. J Ethnopharmacol 189: 361-385, 2016.

32. Lee YS, Ha JH, Yong CS, Lee DU, Huh K, Kang YS, Lee SH, Jung MW and Kim JA: Inhibitory effects of constituents of Gastrodia elata Bl. on glutamate-induced apoptosis in IMR-32 human neuroblastoma cells. Arch Pharm Res 22: 404-409, 1999.

33. Zhao X, Zou Y, Xu H, Fan L, Guo H, Li X, Li G, Zhang X and Dong M: Gastrodin protect primary cultured rat hippocampal neurons against amyloid-beta peptide-induced neurotoxicity via ERK1/2-Nrf2 pathway. Brain Res 1482: 13-21, 2012.

34. Jang JH, Son Y, Kang SS, Bae CS, Kim JC, Kim SH, Shin T and Moon C: Neuropharmacological potential of Gastrodia elata Blume and its components. Evid Based Complement Alternat Med 2015: 309261, 2015.

35. Zhang WJ, Tan YF, Yue JT, Vranic M and Wojtowicz JM: Impairment of hippocampal neurogenesis in streptozotocin-treated diabetic rats. Acta Neurol Scand 117: 205-210, 2008.

36. Li ZG, Zhang W, Grunberger G and Sima AA: Hippocampal neuronal apoptosis in type 1 diabetes. Brain Res 946: 221-231, 2002.

37. Sadeghi A, Hami J, Razavi S, Esfandiary E and Hejazi Z: The effect of diabetes mellitus on apoptosis in hippocampus: Cellular and molecular aspects. Int J Prev Med 7: 57, 2016.

38. Zhuang S and Schnellmann RG: A death-promoting role for extracellular signal-regulated kinase. J Pharmacol Exp Ther 319: 991-997, 2006

39. Murray B, Alessandrini A, Cole AJ, Yee AG and Furshpan EJ: Inhibition of the p44/42 MAP kinase pathway protects hippocampal neurons in a cell-culture model of seizure activity. Proc Natl Acad Sci USA 95: 11975-11980, 1998.

40. Choi BH, Hur EM, Lee JH, Jun DJ and Kim KT: Protein kinase Cdelta-mediated proteasomal degradation of MAP kinase phosphatase-1 contributes to glutamate-induced neuronal cell death. J Cell Sci 119: 1329-1340, 2006.

41. Poitry-Yamate CL, Vutskits L and Rauen T: Neuronal-induced and glutamate-dependent activation of glial glutamate transporter function. J Neurochem 82: 987-997, 2010

42. Stanciu M, Wang Y, Kentor R, Burke N, Watkins S, Kress G, Reynolds I, Klann E, Angiolieri MR, Johnson JW and DeFranco DB: Persistent activation of ERK contributes to glutamate-induced oxidative toxicity in a neuronal cell line and primary cortical neuron cultures. J Biol Chem 275: 12200-12206, 2000.

43. Cagnol S, Van Obberghen-Schilling E and Chambard JC: Prolonged activation of ERK1,2 induces FADD-independent caspase 8 activation and cell death. Apoptosis 11: 337-346, 2006.

44. de Bernardo S, Canals S, Casarejos MJ, Solano RM, Menendez J and Mena MA: Role of extracellular signal-regulated protein kinase in neuronal cell death induced by glutathione depletion in neuron/glia mesencephalic cultures. J Neurochem 91: 667-682, 2004. 
45. Shen GN, Liu L, Feng L, Jin Y, Jin MH, Han YH, Jin $\mathrm{CH}$ Jin YZ, Lee DS, Kwon TH, et al: Knockdown of peroxiredoxin $\mathrm{V}$ increases glutamateinduced apoptosis in HT22 hippocampal neuron cells. Mol Med Rep 17: 7827-7834, 2018.

46. Cheng B and Mattson MP: NT-3 and BDNF protect CNS neurons against metabolic/excitotoxic insults. Brain Res 640: 56-67, 1994

47. Xiong H, Futamura T, Jourdi H, Zhou H, Takei N, Diverse-Pierluissi M, Plevy S and Nawa H: Neurotrophins induce BDNF expression through the glutamate receptor pathway in neocortical neurons. Neuropharmacology 42: 903-912, 2002.

48. Almeida RD, Manadas BJ, Melo CV, Gomes JR, Mendes CS, Graos MM, Carvalho RF, Carvalho AP and Duarte CB: Neuroprotection by BDNF against glutamate-induced apoptotic cell death is mediated by ERK and PI3-kinase pathways. Cell Death Differ 12: 1329-1343, 2005.

49. $\mathrm{Hu} \mathrm{P}$ and Kalb RG: BDNF heightens the sensitivity of motor neurons to excitotoxic insults through activation of TrkB. J Neurochem 84: 1421-1430, 2003.
50. Koh JY, Gwag BJ, Lobner D and Choi DW: Potentiated necrosis of cultured cortical neurons by neurotrophins. Science 268: 573-575, 1995.

51. Glazner GW and Mattson MP: Differential effects of BDNF, ADNF9, and TNFalpha on levels of NMDA receptor subunits, calcium homeostasis, and neuronal vulnerability to excitotoxicity. Exp Neurol 161: 442-452, 2000.

52. El IA and Trenkner E: Growth factors and taurine protect against excitotoxicity by stabilizing calcium homeostasis and energy metabolism. J Neurosci 19: 9459-9468, 1999.

53. Bian L, Bi X, Ai Q, Guo J, Dong S, Xu J, Zhong L and Lu D: Effects of gastrodin on apoptotic factors of cerebral cortex neuron in epileptic rats. Chin J Neuroanat 32: 37-43, 2016.

(i) (3) This work is licensed under a Creative Commons

EY No ND Attribution-NonCommercial-NoDerivatives 4.0 International (CC BY-NC-ND 4.0) License. 